\title{
Mechanisms by which common variants in the TCF7L2 gene increase risk of type 2 diabetes
}

\author{
Valeriya Lyssenko, ${ }^{1}$ Roberto Lupi, ${ }^{2}$ Piero Marchetti, ${ }^{2}$ Silvia Del Guerra, ${ }^{2}$ Marju Orho-Melander, ${ }^{1}$ \\ Peter Almgren, ${ }^{1}$ Marketa Sjögren, ${ }^{1}$ Charlotte Ling, ${ }^{1}$ Karl-Fredrik Eriksson, ${ }^{3}$ Åsa-Linda Lethagen, ${ }^{1}$ \\ Rita Mancarella, ${ }^{2}$ Göran Berglund, ${ }^{3}$ Tiinamaija Tuomi, ${ }^{4,5}$ Peter Nilsson, ${ }^{3}$ \\ Stefano Del Prato, 2 and Leif Groop ${ }^{1,5}$
}

\begin{abstract}
1Department of Clinical Sciences, Diabetes and Endocrinology, and Lund University Diabetes Center, Lund University, Malmö, Sweden. ${ }^{2}$ Department of Endocrinology and Metabolism, Metabolic Unit, University of Pisa, Pisa, Italy. ${ }^{3}$ Department of Clinical Sciences, Faculty of Medicine, Lund University, Malmö, Sweden. ${ }^{4}$ Research Program for Molecular Medicine, Helsinki University, and Genetic Institute, Folkhälsan Research Center, Helsinki, Finland. 5Department of Medicine, Helsinki University Central Hospital, Helsinki, Finland.
\end{abstract}

\begin{abstract}
Genetic variants in the gene encoding for transcription factor-7-like 2 (TCF7L2) have been associated with type 2 diabetes (T2D) and impaired $\beta$ cell function, but the mechanisms have remained unknown. We therefore studied prospectively the ability of common variants in TCF7L2 to predict future T2D and explored the mechanisms by which they would do this. Scandinavian subjects followed for up to 22 years were genotyped for 3 SNPs (rs7903146, rs12255372, and rs10885406) in TCF7L2, and a subset of them underwent extensive metabolic studies. Expression of TCF7L2 was related to genotype and metabolic parameters in human islets. The CT/TT genotypes of SNP rs7903146 strongly predicted future T2D in 2 independent cohorts (Swedish and Finnish). The risk $T$ allele was associated with impaired insulin secretion, incretin effects, and enhanced rate of hepatic glucose production. TCF7L2 expression in human islets was increased 5-fold in T2D, particularly in carriers of the TT genotype. Overexpression of TCF7L2 in human islets reduced glucose-stimulated insulin secretion. In conclusion, the increased risk of T2D conferred by variants in TCF7L2 involves the enteroinsular axis, enhanced expression of the gene in islets, and impaired insulin secretion.
\end{abstract}

\section{Introduction}

Type 2 diabetes (T2D) prevalence is increasing in an epidemic fashion worldwide, with a predicted doubling of the prevalence to 325 million affected persons in 25 years. This increase has been ascribed to a collision between genes and increasing globalization of the Western lifestyle, but few genetic variants have been consistently associated with T2D (1-3). This has now partially changed, as SNPs in the transcription factor-7-like 2 (TCF7L2) gene were reported to be strongly associated with T2D as well as with impaired insulin secretion (4-11). TCF7L2 encodes for a transcription factor involved in Wnt signalling (12). Heterodimerization of TCF7L2 with $\beta$-catenin induces transcription of a number of genes, including intestinal proglucagon (13).

The aims of this study were: (a) to assess whether variants in TCF7L2 could predict future T2D in 2 large prospective cohorts of 9,663 individuals from Sweden (Malmö Preventive Project [MPP]) and Finland (Botnia study) $(14,15)$; and (b) to explore genotypephenotype correlations in a subset of these individuals by studying influence on insulin, glucagon, and glucose-dependent insulinotropic peptide (GIP) levels; incretin effects; as well as effects on hepatic and peripheral insulin sensitivity. In addition, as a haplotype analysis of SNPs in TCF7L2 recently identified haplotype A as

Nonstandard abbreviations used: AIR, acute insulin response; AUC, area under the curve; $D^{\prime}$, linkage disequilibrium; EGP, endogenous glucose production; GIP, glucose-dependent insulinotropic peptide; GLP-1, glucagon-like peptide 1; HOMA, homeostasis model assessment index; IFG, impaired fasting glucose; IGT, impaired glucose tolerance; IVGTT, i.v. glucose tolerance test; MPP, Malmö Preventive Project; OGTT, oral glucose tolerance test; OR, odds ratio; TCF7L2, transcription factor-7-like 2 gene; T2D, type 2 diabetes.

Conflict of interest: The authors have declared that no conflict of interest exists. Citation for this article: J. Clin. Invest. 117:2155-2163 (2007). doi:10.1172/JCI30706. being associated with BMI and leptin levels (16), we also assessed these phenotype-genotype relationships in the Botnia cohort, (c) investigate in vitro in human islets whether risk genotypes influence transcription of TCF7L2 as well as insulin and glucagon secretion. As expression of TCF7L2 was markedly increased in islets from patients with T2D, we also (d) overexpressed TCF7L2 using an adenoviral vector in human islets and related this to level of insulin and glucagon gene expression.

\section{Results}

Common variants in TCF7L2 predict future T2D. Of the 7,061 persons included in the Swedish MPP study, 1,731 (24.5\%) had impaired fasting glucose (IFG) and/or impaired glucose tolerance (IGT), while the remaining 5,330 had normal glucose tolerance at baseline. During the median follow-up period of 22 years, 1,422 individuals developed diabetes (Table 1). The frequencies of the risk CT/TT genotypes of SNP rs7903146 and the GT/TT genotypes of rs12255372 in TCF7L2 were $45.9 \%$ and $48.5 \%$, respectively. These 2 SNPs were in strong linkage disequilibrium $\left(D^{\prime}=0.86\right)$. The frequency of the risk T allele of both SNPs was significantly higher in converters than in nonconverters (rs7903146: $31.3 \%$ versus $25.2 \%, P<0.0001$; rs $12255372: 32.0 \%$ versus $27.1 \%, P<0.0001)$. Carriers of rs7903146 CT/TT genotypes and carriers of rs12255372 GT/TT genotypes had higher risk of future T2D than CC and GG genotype carriers (odds ratio [OR] 1.58, 95\% CI 1.38-1.81, $P<0.0001$, and 1.42, 95\% CI 1.24-1.62, $P<0.0001$, respectively) (Figure 1A; Supplemental Table 1A; supplemental material available online with this article; doi:10.1172/JCI30706DS1). Given that the 2 SNPs basically capture the same genetic information, we hereafter present data only for rs7903146.

In the Botnia prospective study, a subset of the Botnia study, $34.7 \%$ of the 2,651 persons who were diabetes free at baseline car- 
Table 1

Clinical characteristics of study subjects

\begin{tabular}{|c|c|c|c|c|c|}
\hline \multirow[b]{3}{*}{ Number (M/F) } & \multicolumn{2}{|c|}{ Prospective studies } & \multicolumn{3}{|c|}{ Metabolic studies } \\
\hline & \multirow{2}{*}{$\begin{array}{c}\text { MPP } \\
7,061(4,392 / 2,669)\end{array}$} & \multirow{2}{*}{$\begin{array}{c}\text { Botnia study } \\
2,651(1,211 / 1,440)\end{array}$} & \multicolumn{2}{|c|}{ Malmö cohort } & \multirow{2}{*}{$\begin{array}{l}\text { Botnia study } \\
306(149 / 157)\end{array}$} \\
\hline & & & NGT: 94 (73/21) & IGT/T2D: 156 (150/6) & \\
\hline Follow-up period (yr) & $23.7(18.5-27.0)$ & $7.7(5.1-10.4)$ & - & - & - \\
\hline Diabetes converters $(n[\%])$ & $1,422(20.1)$ & $150(5.7)$ & - & - & - \\
\hline Age (yr) & $46.5 \pm 5.8$ & $44.9 \pm 14.2$ & $62.4 \pm 8.5$ & $64.1 \pm 5.9$ & $53.1 \pm 10.9$ \\
\hline $\mathrm{BMI}\left(\mathrm{kg} / \mathrm{m}^{2}\right)$ & $24.5 \pm 3.4$ & $25.6 \pm 4.1$ & $26.1 \pm 3.7$ & $27.4 \pm 3.5$ & $26.6 \pm 4.0$ \\
\hline Fasting glucose (mmol/l) & $5.6 \pm 0.5$ & $5.5 \pm 0.6$ & $5.4 \pm 0.6$ & $8.5 \pm 3.6$ & $5.1 \pm 0.5$ \\
\hline Fasting insulin (mU/l) & $8.9 \pm 9.5$ & $8.0 \pm 5.2$ & $8.0 \pm 4.6$ & $15.3 \pm 11.1$ & $6.4 \pm 5.6$ \\
\hline $\mathrm{HOMA}\left(\mathrm{mmol} \times \mathrm{mU} / \mathrm{I}^{2}\right)$ & $2.2 \pm 2.4$ & $2.0 \pm 1.4$ & $1.6 \pm 1.0$ & $4.8 \pm 3.9$ & $1.5 \pm 1.3$ \\
\hline
\end{tabular}

Data are presented as mean $\pm \mathrm{SD}$ or as median (interquartile range). NGT, normal glucose tolerance.

ried the risk CT/TT genotypes of rs7903146. Similar to the findings in MPP, a higher risk for future T2D was seen in the CT/TT compared with CC genotype carriers (OR 1.61, 95\% CI 1.14-2.27, $P=0.007$; Figure 1B; Supplemental Table 1A).

More recently, a haplotype consisting of 2 SNPs and microsatellite marker DG10S478X has been shown to influence the results (16). DG10S478X can be captured by SNP rs10885406. The combination of allele A of SNP rs 10885406 and allele C of rs7903146 was designated HapA; the combination of alleles C or T of rs7903146 and allele A of rs10885406 HapAB and of allele T of rs7903146 and allele $\mathrm{G}$ of rs 10885406 as HapB (these 2 alleles were in complete linkage disequilibrium) (Supplemental Table 1B). HapA has been suggested to be under selective pressure in Europeans and Asians (16) and associated with obesity (high BMI and leptin levels). We therefore also genotyped SNP rs10885406 in the Botnia cohort. HapA (OR 0.73; $P=0.13$ ) was not associated with increased risk of future T2D (Supplemental Table 1C).

Risk genotypes in TCF7L2 are associated with impaired insulin secretion. In order to delineate the mechanisms by which the risk genotypes in TCF7L2 increased risk of T2D, a number of in vivo and in vitro studies were carried out. The early insulin response to an oral glucose tolerance test (OGTT), also called the insulinogenic index, was assessed at baseline in 1,038 individuals from the MPP cohort. Carriers of the risk CT/TT genotypes $(n=481)$ had lower insulinogenic index than carriers of the CC genotypes $(n=557)(9.3 \pm 5.5$ versus $10.2 \pm 5.3 ; P=0.0006$ ) (Figure 2A; Supplemental Table 2A). No significant difference was observed in insulin sensitivity estimated as homeostasis model assessment index-IR (HOMA-IR) between different genotype carriers $(P=0.3)$. Insulin secretion adjusted for insulin sensitivity, i.e., disposition index, was also lower in CT/TT than in CC genotype carriers $(7.1 \pm 5.5$ versus $8.1 \pm 6.2 ; P=0.005)$ (Figure 2B; Supplemental Table 2A). These results were replicated in participants from the Botnia study: the CT/TT genotype carriers showed reduced insulinogenic index $(4.8 \pm 3.8$ versus $5.5 \pm 4.4$; $P=0.03$ ) as compared with carriers of the CC genotype, with abnormal glucose tolerance (Supplemental Table 2A). Notably, insulin secretion also decreased in $\mathrm{HapAB}$ and to a greater extent in $\mathrm{HapB}$ $(4.9 \pm 3.7$ and $4.7 \pm 4.1)$ compared with HapA hyperglycemic individuals $(5.6 \pm 4.5 ; P=0.03)$ (Supplemental Table 2B). Furthermore, converters carrying the risk $\mathrm{CT} / \mathrm{TT}$ genotypes had a more severe deterioration in insulin secretion (insulinogenic index: coefficient for differences between the slopes, $-0.03, P=0.034$; disposition index: coefficient for differences between the slopes, -0.05 ; $P=0.003$ ) as compared with the CC genotypes (Figure 2C).
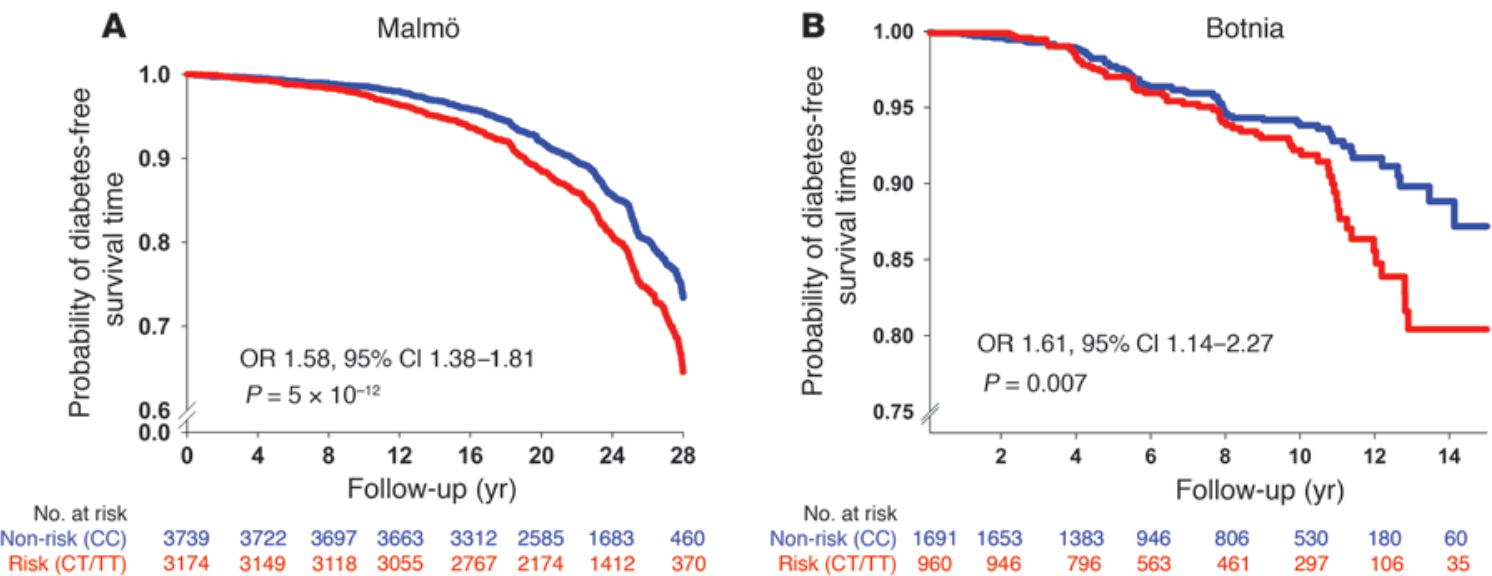

$\begin{array}{lllllllll}\text { Non-risk (CC) } & 3739 & 3722 & 3697 & 3663 & 3312 & 2585 & 1683 & 460 \\ \text { Risk (CT/TT) } & 3174 & 3149 & 3118 & 3055 & 2767 & 2174 & 1412 & 370\end{array}$

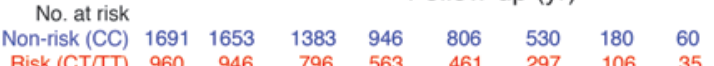

Figure 1

Diabetes-free survival (Kaplan-Meier curves) in carriers of different TCF7L2 rs7903146 genotypes. (A) Diabetes-free survival in the MPP $(n=6,913)$. (B) Diabetes-free survival in the Botnia study $(n=2,651)$. The blue lines represent carriers of non-risk CC genotype; the red lines represent carriers of the risk CT/TT genotypes. $95 \%$ Cls are from logistic regression analyses adjusted for age at entry and time of follow-up, $\mathrm{BMI}$, sex, and family history of diabetes. 


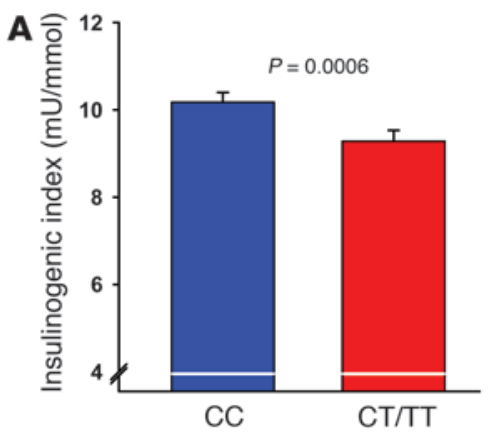

C

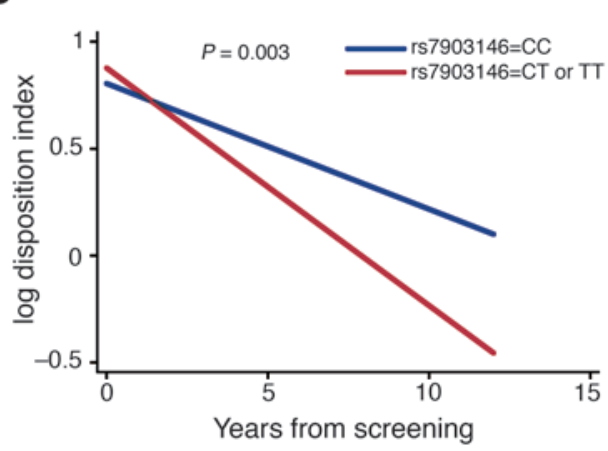

E

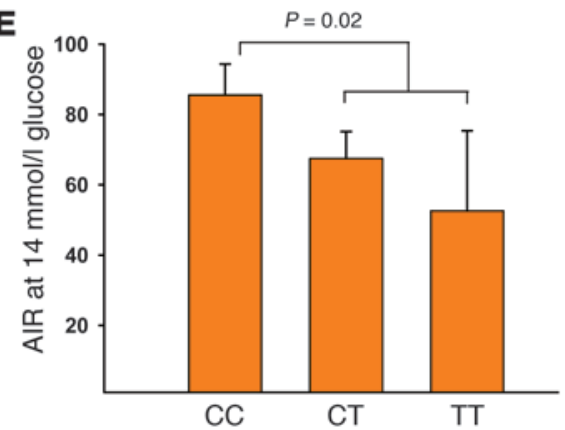

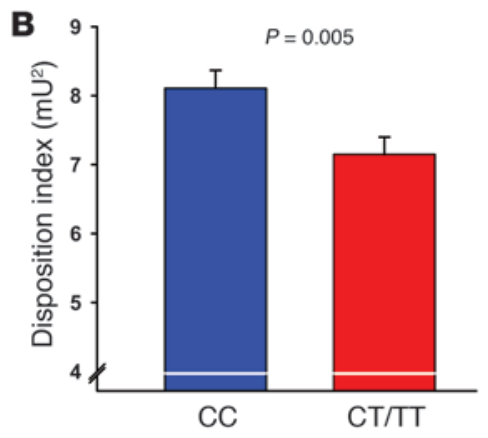

D

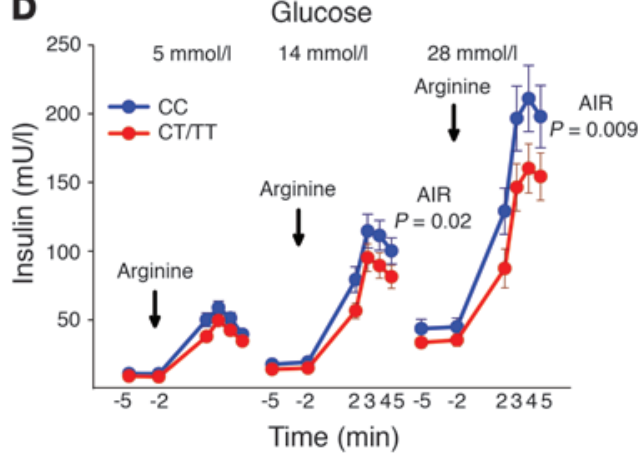

F

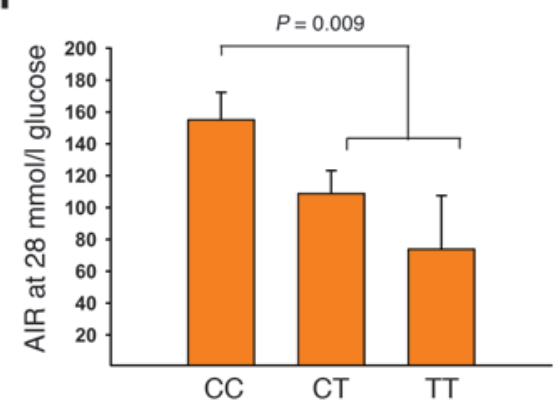

Figure 2

Insulin secretion according to different TCF7L2 rs7903146 genotypes or haplotypes. (A) Insulinogenic index, i.e., incremental (40-minute) insulin response to oral glucose (Malmö cohort; $n=1,038$ ). (B) Disposition index represents the insulinogenic index adjusted for insulin sensitivity by the HOMA index (Malmö cohort; $n=1,038$ ). (C) Change in insulin secretion (disposition index) over time in subjects who converted to T2D (Botnia cohort, $n=120 ; n=420$ observations). (D) AIR to arginine at basal level ( $5 \mathrm{mmol} / \mathrm{l})$ and 14 and $28 \mathrm{mmol} / \mathrm{l}$ of glucose according to TCF7L2 rs7903146 genotypes in subjects with IGT/T2D (Malmö cohort; $n=130$ ). (E) AIR to arginine at $14 \mathrm{mmol} / \mathrm{l}$ of glucose according to TCF7L2 rs7903146 genotypes in subjects with IGT/T2D. (F) AIR to arginine at $28 \mathrm{mmol} / \mathrm{l}$ of glucose according to TCF7L2 rs7903146 genotypes in subjects with IGT/ T2D. Bars represent mean \pm SEM. Blue lines represent non-risk and red lines risk genotype carriers of rs7903146 in TCF7L2.
We also assessed influence of SNPs in TCF7L2 on arginine-stimulated insulin secretion in a subset of 250 subjects from the MPP cohort $(17,18)$. The acute insulin response (AIR) to arginine was significantly reduced at both $14 \mathrm{mmol} / \mathrm{l}(67 \pm 60$ versus $86 \pm 70$ $\mathrm{mU} / \mathrm{l} ; P=0.02)$ and $28 \mathrm{mmol} / \mathrm{l}(107 \pm 113$ versus $155 \pm 138 \mathrm{mU} / \mathrm{l}$; $P=0.009)$ of glucose in the CT/TT versus CC genotype carriers with abnormal glucose tolerance (i.e., with IFG/IGT and T2D) (Figure 2, D-F), while no difference was seen between different genotype carriers with normal glucose tolerance (Supplemental Table 3). The slope of potentiation of insulin secretion by glucose, which was used as a measure of glucose sensitivity of the $\beta$ cells (19), was not significantly different in the 2 genotype groups.

Risk genotypes in TCF7L2 are associated with an impaired incretin effect. The incretin concept refers to a better glucose-dependent insulin response to oral than to i.v. glucose due to additional stimulation of insulin secretion by intestinal insulin secretagogues such as glucagon-like peptide 1 (GLP-1) and GIP (20). The incretin effect is therefore confined to situations of elevated plasma glucose levels. Given that TCF7L2 has been proposed to bind to the promoter of the proglucagon gene, we compared insulin response with oral and i.v. glucose in 403 individuals using both OGTTs and i.v. glucose tolerance tests (IVGTTs) to obtain a biological measure of the incretin effect. In support of a defective enteroinsular axis, there was a $20 \%$ lower insulin response to oral than to i.v. glucose in the risk CT/TT than in the CC genotype carriers, with fasting glucose of at least $5.5 \mathrm{mmol} / \mathrm{l}$ (AUC insulin OGTT/ $_{\text {/ }}$ $\mathrm{AUC}_{\text {insulin IVGTT }}$ [where AUC is area under the curve and ins is insulin], $5.2 \pm 1.9$ versus $6.7 \pm 3.2, P<0.0001$; incretin effect with $100 \% \times\left(\mathrm{AUC}_{\text {ins OGTT }}-\mathrm{AUC}_{\text {ins IVGTT }}\right) / \mathrm{AUC}_{\text {ins OGTT }}, 78.3 \pm 7.9$ versus $81.8 \pm 8.9, P=0.003$ ) (21) (Figure 3, A and B, and Supplemental Table 4). Additionally, carriers of $\mathrm{HapB}$ had a more severe incretin defect than carriers of HapAB and HapA (AUC insulin OGTT/ $_{4}$ $\mathrm{AUC}_{\text {insulin IVGTT }} 5.0 \pm 1.8,5.4 \pm 2.0$, and $6.7 \pm 3.2, P=0.0003$; incretin effect, $77.3 \pm 8.2,79.1 \pm 8.0$, and $81.8 \pm 8.6, P=0.004$ ) (Supplemental Table 4). The correlation coefficients the insulin response to oral and i.v. glucose were significantly different between risk and non-risk genotype carriers ( $r=0.68$ versus $r=0.52 ; P=0.007$ ), again suggesting an impaired incretin effect (Figure 3A). This correlation was strongest in $\mathrm{HapB}$ carriers with fasting hyperglycemia $(r=0.72 ; P<0.0001)$. We also tested the same correlation using a different gene variant, the Pro12Ala polymorphism in PPARG, with no presumed incretin effect (15). There was no difference in the slopes relating insulin responses to oral and i.v. glucose between carriers of this polymorphism (Figure 1A). 

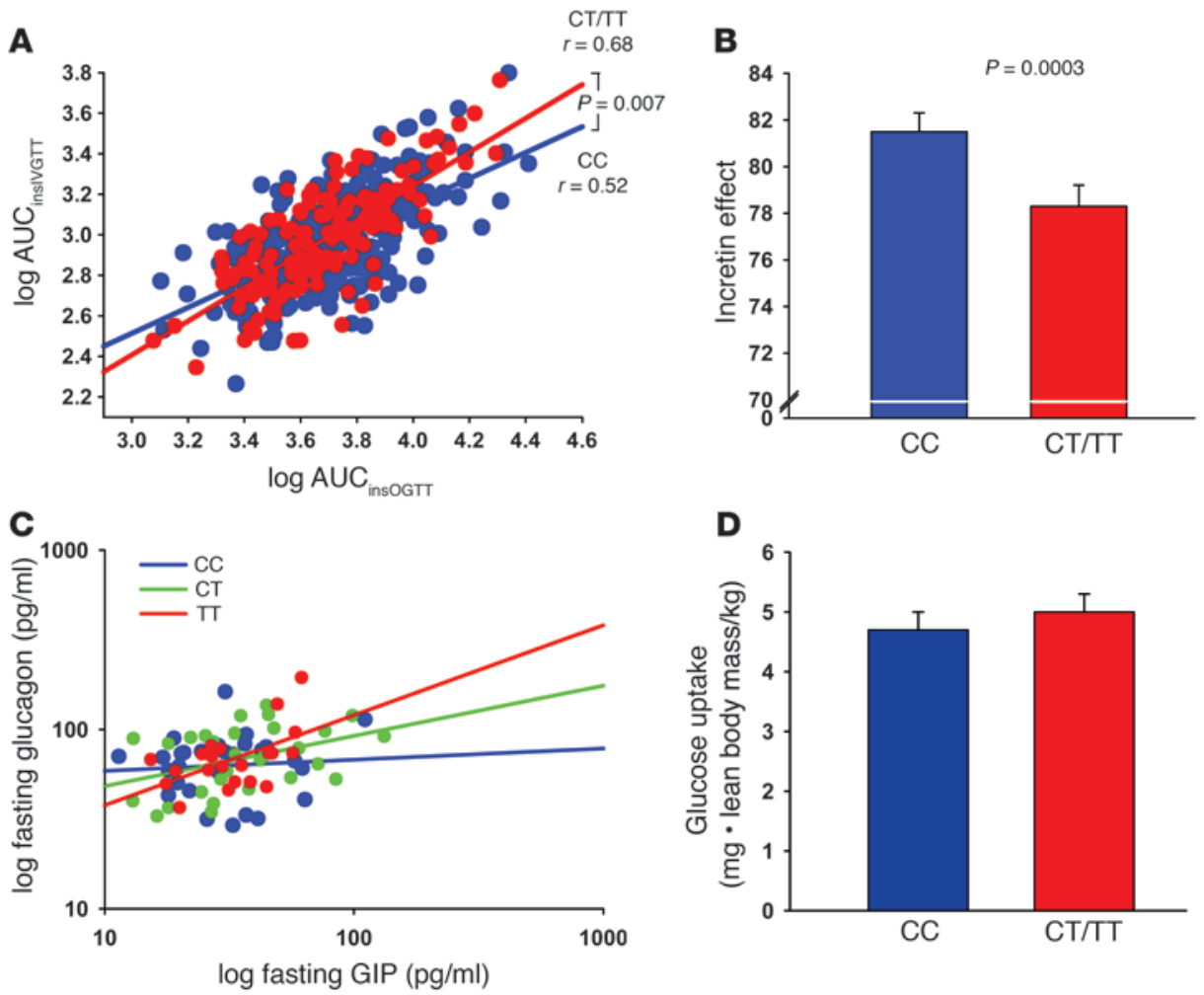

Figure 3

Metabolic effects of different genotypes in rs7903146 of TCF7L2. (A) Correlation between the area under the insulin curve during OGTT and IVGTT (Botnia cohort; $n=403$ ). (B) Incretin effect measured as $100 \% \times\left(A C_{\text {ins }}\right.$ OGTT $\left.-A U C_{\text {ins IVGTT }}\right) /$ AUC $_{\text {ins OGTT in hyperglycemic subjects }}$ (fasting plasma glucose $>5.4 \mathrm{mmol} / \mathrm{l}$ ) (Botnia cohort; $n=235$ ). (C) Correlation between fasting GIP $(\mathrm{pg} / \mathrm{ml})$ and glucagon $(\mathrm{pg} / \mathrm{ml})$ concentrations (Botnia cohort; $n=78$ ). (D) Whole-body glucose uptake during a euglycemic-hyperinsulinemic clamp. (E) Basal EGP. (F) Change in BMI over time in subjects who converted to T2D. Bars represent mean \pm SEM. Blue lines represent nonrisk and red lines risk genotype carriers of rs7903146 in TCF7L2.

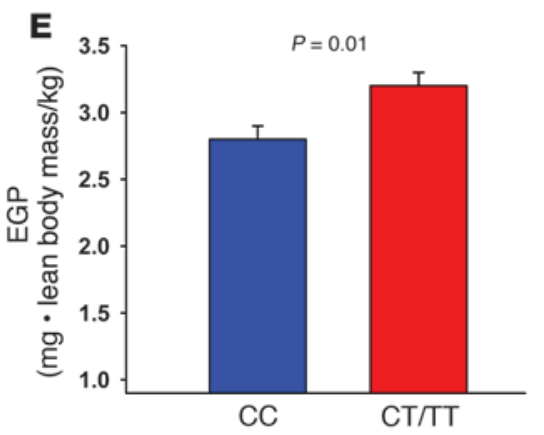

To explore which incretin hormones would be involved, we attempted to measure plasma concentrations of the 2 incretin candidates, GLP-1 and GIP, as well as of glucagon. Unfortunately, we were not able from the stored samples to measure GLP-1, so the analyses were restricted to GIP and glucagon. However, preliminary data (22) suggest that there is no decrease in GLP-1 levels in carriers of risk genotypes in TCF7L2. While GLP-1 is known to stimulate insulin and inhibit glucagon secretion, GIP stimulates both insulin and glucagon secretion. We did not observe any difference in plasma glucagon or GIP concentrations between different genotype carriers (Supplemental Table 5). As expected, fasting GIP and glucagon concentrations correlated positively in all individuals $(r=0.32 ; P=0.004 ; n=78)$, but this correlation was due to the relationship seen in TT genotype carriers $(r=0.59 ; P=0.009 ; n=21)$ (Figure 3C), while there was no significant correlation between GIP and glucagon concentrations in CC carriers $(r=0.05 ; P=0.82)$. No significant correlation was seen between 2-hour GIP and glucagon concentrations. However, 2-hour GIP and insulin concentrations correlated in the whole cohort $(r=0.34$; $P=0.003 ; n=77)$ and in CC $(r=0.55 ; P=0.004 ; n=28)$ but not in TT carriers $(r=0.35 ; P=0.2 ; n=21)$ (Supplemental Figure 1B).
Risk genotypes in TCF7L2 are associated with hepatic but not peripheral insulin resistance. Whole-body glucose uptake did not differ between the risk and non-risk genotype carriers of TCF7L2 (Figure $3 \mathrm{D}$ and Supplemental Table 3). However, the rate of endogenous glucose production (EGP) was significantly higher in the CT/TT than CC genotype carriers $(3.2 \pm 0.8$ versus $2.8 \pm 0.3 \mathrm{mg} / \mathrm{kg}$ lean body/min; $P=0.01)$, suggesting impaired suppression of basal hepatic glucose production (Figure 3E and Supplemental Table 3). In line with findings on insulin secretion, there was stepwise increase in EGP in HapA, HapAB, and HapB individuals $(P=0.03$ for HapA versus HapB) (Supplemental Table 3).

Risk genotypes in TCF7L2 are associated with BMI. As SNPs in TCF7L2, particularly the HapA haplotype, have been suggested to influence BMI and leptin levels (16), we also analyzed whether risk genotypes and different haplotypes influence these variables. While there was no association between risk genotypes in SNP rs7903146 and BMI in the Malmö cohort, in the Botnia study, homozygous carriers of TT genotypes had lower BMI (TT: $24.2 \pm 3.5$, CT: $25.9 \pm 4.0$, CC: $25.6 \pm 4.2 ; P=0.004$ ) (Supplemental Table 2A). 

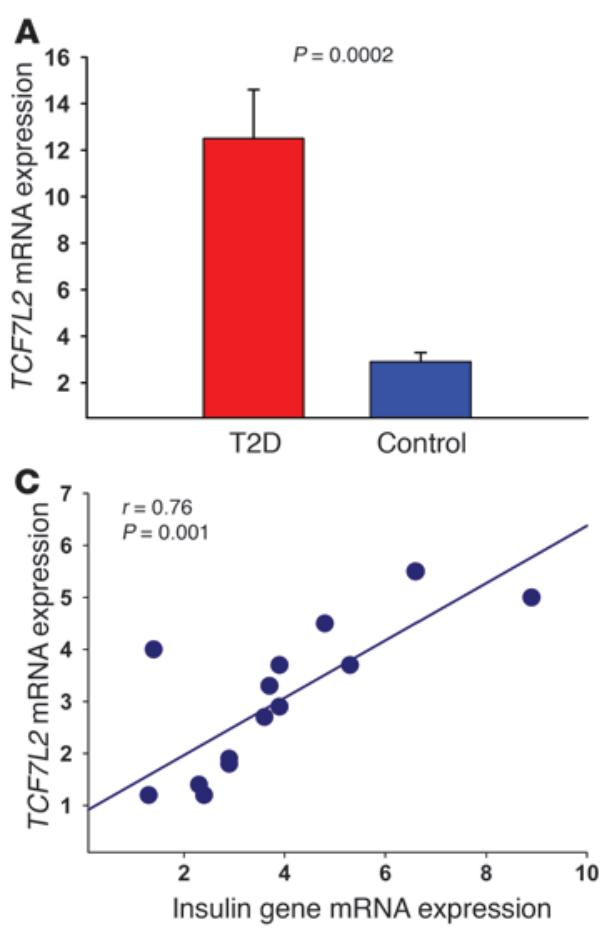
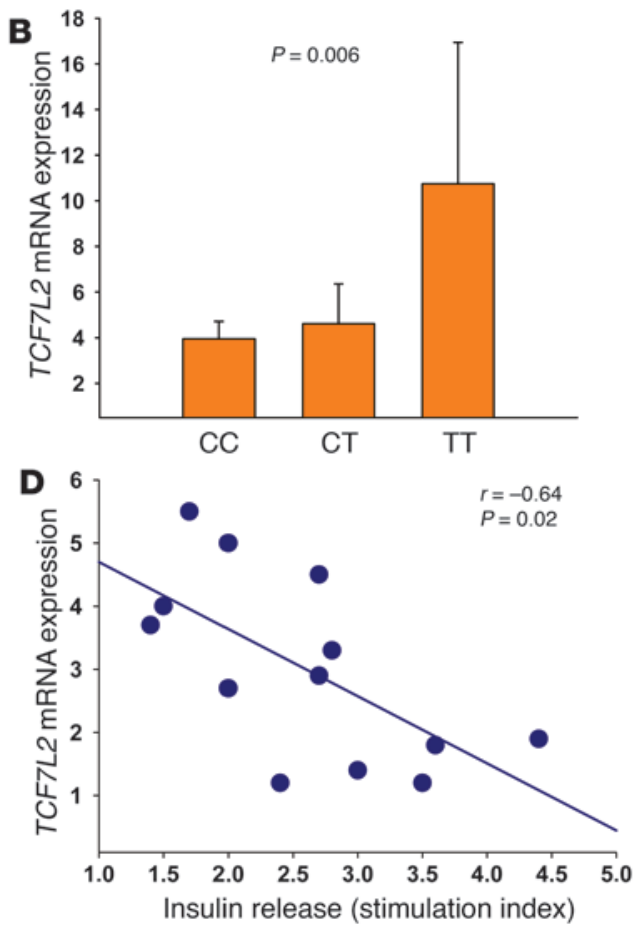

\section{Figure 4}

Expression of TCF7L2 in human pancreatic islets was influenced by T2D and correlates positively with insulin gene expression and negatively with insulin secretion. (A) The TCF7L2 mRNA levels in human pancreatic islets. (B) The TCF7L2 mRNA levels in different TCF7L2 rs7903146 genotype carriers. ( $P$ refers to ANCOVA comparison between $\mathrm{CC}, \mathrm{CT}$, and TT genotypes) (C) Correlation between the TCF7L2 mRNA levels and insulin gene expression. (D) Correlation between the TCF7L2 mRNA levels and glucose-stimulated insulin release (stimulation index). Bars represent mean \pm SEM.
Of note, the BMI of converters carrying CT/TT genotypes increased over time (coefficient of differences between the slopes, $0.17, P=0.035$ ) as compared with converters with CC genotypes. None of the haplotypes significantly influenced BMI, in either the Malmö or Botnia cohorts.

There was no significant association between risk genotypes or haplotypes and leptin levels in the Botnia study (Supplemental Table 2, A and B).

Risk genotypes in TCF7L2 increase gene expression in buman islets. Since the SNPs in TCF7L2 are in noncoding regions, we hypothesized that one potential mechanism by which they could exert the observed effects is by influencing expression of TCF7L2 in pancreatic islets. For this purpose we measured TCF7L2 mRNA in pancreatic islets from 7 T2D and 15 nondiabetic human cadaveric organ donors. In the basal state, isolated islets from patients with T2D showed reduced amounts of insulin and increased amounts of glucagon secreted compared with nondiabetic donors $(209 \pm 9$ versus $2,534 \pm 105 \mu \mathrm{U} / \mathrm{ml} / 24 \mathrm{~h}, P=0.0002 ; 54.6 \pm 3.1$ versus $7.5 \pm 0.4 \mathrm{pg} / \mathrm{ml} / 24 \mathrm{~h}, P=0.0002)$. The mRNA level of TCF7L2 was 5 -fold higher in human pancreatic islets from $\mathrm{T} 2 \mathrm{D}$ than nondiabetic donors $(12.5 \pm 2.1$ versus $2.9 \pm 0.4 ; P=0.0002$; Figure $4 \mathrm{~A})$, and the expression increased with number of $\mathrm{T}$ alleles in the whole cohort ( $P=0.006$ adjusted for diabetes status; Figure $4 \mathrm{~B}$ ). The increase in TCF7L2 expression with increasing numbers of $\mathrm{T}$ alleles was not due to diabetes per se, as it was also seen in the nondiabetic individuals (CC: $3.3 \pm 0.4$; CT: $2.2 \pm 0.7$; TT: $4.6 \pm 0.9$; $P=0.02)$. Expression of TCF7L2 in human islets was lowest in carriers of HapA, intermediate in HapAB carriers, and highest in HapB carriers $(P=0.02)$. Costaining of TCF7L2 with RhodZin (Invitrogen) showed localization of TCF7L2 to $\beta$ cells (Figure 5). Although the expression of the insulin gene was markedly decreased in islets from T2D subjects compared with nondiabetic donors $(0.5 \pm 0.1$ versus $3.8 \pm 0.5 ; P=0.003)$, there was a positive correlation between the mRNA levels of TCF7L2 and insulin gene $(r=0.76 ; P=0.001)$ (Figure $4 \mathrm{C})$; this correlation was strongest in nondiabetic CT/TT genotype carriers $(r=0.81 ; P=0.01)$. Despite this, the TCF7L2 mRNA correlated inversely with glucose-stimulated insulin release (stimulation index) after normalization for total insulin content $(r=-0.64 ; P=0.02)$ (Figure 4D and Supplemental Table 6), particularly in carriers of the CT/TT genotypes $(r=-0.83 ; P=0.04)$, suggesting that TCF7L2 might influence posttranscriptional events of insulin expression.

Having seen the elevated expression of TCF7L2 in T2D islets and its association with impaired insulin secretion, we attempted to experimentally confirm the cross-sectional finding by overexpressing TCF 72 in human islets and relating this to measures of insulin and glucagon secretion using an adenovirus system. This resulted in a 2 -fold overexpression of TCF7L2 in human islets, which, again was associated with increased insulin gene expression $(2.7 \pm 0.4$ versus $1 ; P<0.01)$ and reduced glucose-stimulated insulin secretion $(0.03 \pm 0.02$ versus $0.06 \pm 0.02 ; P<0.05)$ compared with expression in control islets overexpressing only the vector. These data thus provide evidence that overexpression of TCF7L2 in human islets alters insulin but not glucagon secretion.

\section{Discussion}

The key finding from these studies is that the T allele of SNP rs7903146 of TCF7L2 strongly predicts future T2D and is associated with enhanced expression of TCF7L2 in human islets as well as with impaired insulin secretion both in vitro and in vivo. The risk T allele of this SNP also seems to have effects on the enteroinsular axis and the relationship between the incretin hormone GIP and its target hormones, glucagon and insulin.

In all whole-genome SNP scans, TCF7L2 was among the genes most strongly associated with T2D (23-27) (http://www.broad. mit.edu/diabetes). Also, in the Diabetes Prevention Project (DPP) study, common variants in TCF7L2 predicted future T2D in the placebo group but not in the intervention groups with lifestyle modi- 

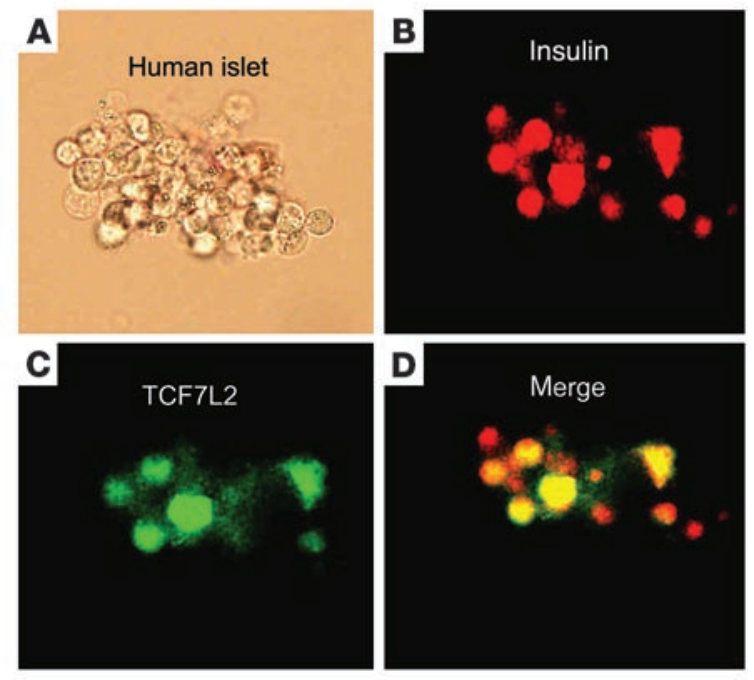

\section{Figure 5}

Colocalization of TCF7L2 expression with insulin in pancreatic islets(A-D). Double staining of RhodZin (red) (B) and TCF7L2 (green) (C) in human pancreatic islets (yellow) (D).

fication or metformin (6). The results from our large prospective observational studies are very similar to the results in the observational placebo arm of DPP. The mechanism(s) by which risk alleles in TCF7L2 increase risk of T2D most likely involve impairment of insulin secretion. This is consistent with findings from most (6-8) but not all previous studies (28). The present study may shed some new light on these discrepancies. We observed in multiple substudies an impairment in both glucose and arginine-stimulated insulin secretion in carriers of risk alleles of TCF7L2. The risk CT/TT genotypes were also associated with a consistent impairment in insulin response to oral glucose over time in individuals who converted to diabetes. It has been suggested that refinement of the TCF7L2 locus into HapA and HapB by genotyping an additional SNP, rs10885406, would improve the results. However, the effect is marginal and restricted to heterozygous carriers of rs7903146 (CT), among whom GG carriers of rs 10885406 have $20 \%$ lower insulin secretion and slightly higher risk of T2D than carriers with AG of rs10885406 (Supplemental Figure 2).

TCF7L2 encodes for a transcription factor involved in Wnt signaling (13). Heterodimerization of TCF7L2 with $\beta$-catenin induces transcription of a number of genes, including those coding for intestinal proglucagon (the prohormone of glucagon) and GLP-1 and -2 (29). In T2D, secretion of GLP-1 is impaired, while that of GIP normal (30). In contrast, the effect of GLP-1 on insulin secretion is maintained, while the effect of GIP on insulin secretion is blunted (30). Also, suppression of glucagon by glucose is impaired in T2D (31).

A normal incretin effect would imply a stronger insulin response to oral than to i.v. glucose. Intriguingly, carriers of the risk T allele of SNP rs7903146 showed a weaker response to oral than to i.v. glucose, suggesting a defective enteroinsular axis. In addition, this impairment was strongest in carriers of the HapB haplotype. In keeping with the glucose-dependent effect of incretins, this effect was restricted to the hyperglycemic state. The impaired incretin effect could be due to both impaired secretion and action of incretin hormones. Although, the fasting concentrations of GIP and glucagon were normal in carriers of the risk genotypes, the correlation between GIP and glucagon was stronger in carriers of the risk T allele. Interestingly, GIP levels correlated with insulin levels at 2 hours of the OGTT in CC but not in TT genotype carriers. These findings could suggest that in carriers of the T allele, GIP has a stronger stimulatory effect on glucagon secretion but a weaker effect on insulin secretion than in carriers of the $\mathrm{C}$ allele. Unfortunately, we were not able to measure GLP-1 concentrations in our stored plasma samples, but preliminary studies suggest that GLP-1 secretion is normal in carriers of the risk T allele (22). Taken together, the data suggest an impaired incretin effect in carriers of the risk genotypes of TCF7L2.

Risk genotype carriers also showed an enhanced rate of EGP, which is primarily due to glucose production by the liver. This could be a consequence of both impaired insulin and enhanced glucagon secretion. However, as circulating glucagon concentrations did not differ between different genotype carriers, this is most likely a consequence of the difference in insulin concentrations observed.

To further explore the mechanisms by which variants in TCF7L2 could impair insulin secretion, we studied genotype-phenotype correlations in human islets. Expression of TCF7L2 was 5-fold higher in islets from patients with T2D than in islets from nondiabetic cadaveric donors. More importantly, nondiabetic carriers of the TT genotype had the highest expression of TCF7L2 in islets. These data are consistent with findings in rodent models for T2D; in both the GK (32) and in the ZDF rat (33), the expression of TCF7L2 is increased in diabetic islets. Our finding of markedly increased expression of TCF7L2 in human pancreatic islets is opposite to a recent report of decreased expression of TCF7L2 in adipose tissue from obese T2D patients (10). Although we do not know the reason for this difference, there might be tissue-specific differences in expression of TCF7L2. Given the high expression of TCF7L2 observed in islets from T2D patients, we cannot exclude additional secondary effects of the diabetic state on gene expression. Cauchi et al. (10) have reported expression of TCF7L2 in human $\beta$ cells purified by FACS. In our study of human islets, TCF7L2 also costained with insulin in $\beta$ cells. Of note, expression of TCF7L2 correlated strongly and positively with expression of the insulin gene but negatively with glucose-stimulated insulin secretion. This paradoxical relationship was particularly strong in carriers of the risk genotype. No relationship was seen between TCF7L2 and glucagon expression in human islets. There is limited information on the relationship between insulin gene expression and stimulated insulin secretion in humans. One possible explanation would be that the increase in insulin gene expression is compensatory to a posttranscriptional defect in insulin secretion, which could involve multiple steps in glucose and ATP-stimulated insulin secretion including exocytosis.

To explore whether the observed correlations between TCF7L2 expression and insulin gene expression and insulin secretion were primary or secondary to the diabetic state, we overexpressed TCF7L2 in nondiabetic human islets using an adenovirus system. In support of a primary effect, a 2-fold elevated expression of TCF7L2 resulted in 2.3-fold increased insulin gene expression and impaired glucose-stimulated insulin secretion, with no effect on glucagon gene expression.

Wnt signaling has recently been shown to regulate pancreatic $\beta$ cell proliferation (34). Addition of Wnt3a to cultured $\beta$ cells and conditional overexpression of $\beta$-catenin led to increased $\beta$ cell proliferation in vitro. Since interaction between TCF7L2 and $\beta$-catenin is a prerequisite for transcription of target genes, it will be important to examine whether TCF7L2 also affects $\beta$ cell proliferation. 
In conclusion, genetic variants in TCF7L2 confer a strong risk of future T2D, possibly by influencing the expression of TCF7L2 in pancreatic islets. Enhanced expression of TCF7L2 reduces insulin but not glucagon secretion. The precise mechanisms by which alterations in TCF7L2 expression relate to impaired insulin secretion as well as the potential involvement of impaired incretin effects require further studies. The fact that TCF7L2 is the gene most strongly associated with T2D susceptibility emphasizes the importance of exploring the potential of manipulating this pathway in future treatment of the disease.

\section{Methods}

\section{Study populations}

In the MPP 33,346 citizens (22,444 men and 10,902 women; mean age 49 years; $24.5 \%$ with IFG and/or 2-hour IGT) from Malmö in southern Sweden participated in a health screening during 1974-1992 (with a 70\% attendance rate) (14). All individuals underwent a physical examination and determination of fasting blood glucose levels, while 19,000 consecutive persons also had an OGTT. Information on lifestyle factors and medical history was obtained via a questionnaire. Of the individuals participating in the initial screening, 22,000 ( $70 \%$ of eligible individuals) were invited to a rescreening visit starting in 2002, during which fasting samples were obtained for measurements of plasma glucose and lipids. To date, 11,145 persons have been restudied; of them 1,422 had developed T2D. In the current study, we included the first 7,061 consecutive individuals with DNA extracted (Table 1), which included all 1,422 patients who had developed T2D.

Insulin measurements were taken for 1,038 of these patients (951 men and 87 women) during the baseline OGTT.

A subgroup of 203 men with IGT at screening visit selected from the MPP study participated 20 years later in more extensive metabolic studies, including a new OGTT, a euglycemic-hyperinsulinemic clamp combined with indirect calorimetry and infusion of $\left[3-{ }^{3} \mathrm{H}\right]$ glucose, and a glucosedependent arginine stimulation test $(17,35,36)$. The men were similar in age but had varying degrees of glucose tolerance; 69 were in the normal range, 52 had IFG and/or IGT, and 82 had T2D. T2D patients were either treated with diet alone (42\%) or with oral hypoglycemic agents, which were withheld the day before the test. In addition, data from 47 subjects (normal glucose tolerance, $n=34$ and T2D, $n=13$ ) who had participated in a glucose-dependent arginine stimulation test were added to sample for assessment of AIR to arginine (18).

The study in Botnia on the West coast of Finland begun in 1990, aiming at identification of genes increasing susceptibility to T2D in members of families with T2D (37). The prospective part included 2,651 nondiabetic family members and/or their spouses (1,211 men and 1,440 women; mean age 45 years) with at least 2 visits during a 7.7-year (median) follow-up period (Table 1) (15). Of them, 150 individuals developed T2D during this period. All subjects participated in a 75-g OGTT after a 12-hour overnight fast. For 2,399 of them, insulin values at the baseline visit were available.

Data from 403 nondiabetic individuals (190 men, 213 women; mean age 47 years) who had participated in both an OGTT and IVGTT no more than 6 months apart were used to study potential incretin effects of genotypes.

Plasma samples from 306 individuals with normal glucose tolerance from a new population-based cohort in the Botnia region was used to measure glucagon concentrations. For this study approximately $10 \%$ of the population aged $18-74$ years (mean age $51 \pm 17$ years) has been invited; the total number of individuals will be approximately 5,000. All participants gave informed consent, and the Institutional Review Board in Vaasa Central Hospital, Vassa, Finland; Lund University; Helsinki University; and the University of Pisa approved the studies.
Multiorgan pancreas donors. Human pancreatic islets were obtained from 19 nondiabetic ( 13 men and 6 women; age [mean \pm SEM] $59 \pm 5$ years; BMI $26 \pm 0.5$ weight $/$ height $^{2}$ ) and 7 T2D ( 4 men and 3 women; age $68 \pm 4$ years; BMI $28 \pm 1.2$ weight $/$ height $\left.^{2}\right)$ multiorgan cadaveric donors with the approval of the Ethics Committee at the University of Pisa, Italy $(38,39)$.

\section{Measurements}

The participants' weight, height, waist and hip circumference, fat-free mass (Botnia study), and blood pressure were measured as previously reported (37). In the MPP cohort at baseline, blood samples were drawn at 0,40 , and 120 minutes of the 75-g OGTT for measurements of blood glucose and serum insulin concentrations, while fasting samples were drawn at followup visits for measurement of plasma glucose and lipid concentrations using standard techniques (36). In the Botnia study, blood samples were drawn at $-10,0,30,60$, and 120 minutes of the OGTT and at $-10,0,2,4,6,8,10,20$, $30,40,50$, and 60 minutes of the IVGTT for measurement of plasma glucose and serum insulin concentrations. Insulin resistance was estimated according to the HOMA as the product of fasting glucose ( $\mathrm{mmol} / \mathrm{l})$ and insulin ( $\mathrm{mU} / \mathrm{l}$ ) divided by the constant 22.5 (40). $\beta$ Cell function was estimated as the insulinogenic index (30 or 40 minutes in MPP) (insulin 30 minutes - fasting insulin / glucose 30 minutes) and the disposition index (insulinogenic index / HOMA), which is a measure of $\beta$ cell function corrected for insulin resistance $(41)$. The total insulin $(\mathrm{mU} / \mathrm{l})$ and glucose $(\mathrm{mmol} / \mathrm{l})$ areas during OGTT were calculated using the trapezoidal rule.

Whole-body insulin sensitivity and basal endogenous glucose production (EGP) in the subgroup of 203 men were measured by a standard 2 h euglycemic-hyperinsulinemic clamp combined with infusion of $\left[3-{ }^{3} \mathrm{H}\right]$ glucose and indirect calorimetry (17).

To quantify $\beta$ cell function, the glucose-dependent arginine stimulation test was used. An acute bolus of arginine was given at 3 different glucose concentrations: fasting and 14 and $28 \mathrm{mmol} / \mathrm{l}$. The AIR to arginine was calculated from the +2 - to 5 -minute values, and glucose potentiation of insulin secretion was calculated as the slope between AIR at fasting and $14 \mathrm{mmol} / \mathrm{l}$ of glucose and used as a measure of the sensitivity of the $\beta$ cell to glucose (17).

A stronger insulin response to oral than to i.v. glucose was used as a measure of an incretin effect. This effect was estimated as the ratio between total insulin responses during OGTT and IVGTT (AUC $\mathrm{Ans}_{\text {OGTT }}$ / $\mathrm{AUC}_{\text {ins IVGTT }}$ ) as well as using a validated formula that estimates the percentage of the differences in insulin responses between oral and i.v. glucose $\left[100 \% \times\left(\mathrm{AUC}_{\text {ins OGTT }}-\mathrm{AUC}_{\text {ins IVGTT }}\right) / \mathrm{AUC}_{\text {ins OGTT }}\right](21)$.

Plasma glucose was measured by hexokinase (MPP) or glucose oxidase (Botnia study) methods. Plasma insulin concentrations were measured by an ELISA assay (Dako) (Botnia study) or by a local radioimmunoassay (MPP) (42); glucagon concentrations were measured by radioimmunoassay (Linco).

Genotyping. Patient DNA was extracted from whole blood using QIAGEN maxipreps. Genotyping was performed by primer extension of multiplex products with detection by matrix-assisted laser desorption ionization-time of flight mass spectroscopy using a SEQUENOM platform (43) for rs7903146 and rs12255372, and for rs10885406 using an allelic discrimination assay-by-design method on ABI 7900 (Applied Biosystems). The combination of allele $C$ of rs7903146 and allele A of rs 10885406 was designated HapA; the combination of allele C or T from rs7903146 and allele A from rs10885406 HapAB and allele T of rs7903146 and allele G of rs10885406 as HapB. Of note, the T allele of rs7903146 and G allele of rs10885406 are in almost complete linkage disequilibrium $\left(D^{\prime}=0.99\right.$; $r^{2}=1$ ). We achieved an average genotyping success rate of $98 \%$ and concordance rate of $99 \%$. DNA extraction and genotyping were performed at the SWEGENE Resource Center for Profiling Polygenic Diseases.

Human pancreatic islets. Isolated pancreatic islets were prepared by collagenase digestion and density gradient purification $(38,39)$. After isolation, 
islets were cultured free-floating in M199 culture medium (Sigma-Aldrich) at $5.5 \mathrm{mmol} / \mathrm{l}$ glucose concentration. Within 3 days of isolation, 24-hour basal insulin and glucagon release was evaluated in the media by Immuno Radiometric assay and RIA techniques, respectively (Pantec and EuroDiagnostica), and the islets were used for RNA extraction. Insulin secretion studies were performed by the batch incubation method. In the batch incubation experiments, following a 45 -minute preincubation period at $3.3 \mathrm{mmol} / 1$ glucose, groups of approximately 30 islets of comparable size were kept at $37^{\circ} \mathrm{C}$ for 45 minutes in Krebs-Ringer bicarbonate solution (KRB), $0.5 \%$ albumin, $\mathrm{pH} 7.4$, containing $3.3 \mathrm{mmol} / \mathrm{l}$ glucose. At the end of this period, medium was completely removed and replaced with KRB containing either 3.3 or $16.7 \mathrm{mmol} / \mathrm{l}$ glucose. After an additional 45 -minute incubation, medium was removed. Samples $(500 \mu \mathrm{l})$ from the different media were stored at $-20^{\circ} \mathrm{C}$ until insulin or glucagon concentrations were measured. The results are expressed as single values or stimulation index (ratio of stimulated release to basal release).

Total RNA was extracted from the islets using the RNeasy Protect Mini Kit (QIAGEN) and quantified by absorbance in $\mathrm{nm}$ at $\mathrm{A}_{260} / \mathrm{A}_{280}$ (to ensure integrity of RNA, the ratio between absorbance at $260 \mathrm{~nm}\left[\mathrm{~A}_{260}\right]$ and absorbance at $280 \mathrm{~nm}\left[\mathrm{~A}_{280}\right]$ was higher than 1.65) in a PerkinElmer spectrophotometer, and its integrity was assessed after electrophoresis in $1.0 \%$ agarose gels by ethidium bromide staining. Human TCF7L2, insulin, and glucagon gene mRNA levels were quantified by reverse transcription reaction followed by real-time quantitative RT-PCR (39). Gene-specific probes and primer pairs for TCF7L2 (Assay-on-Demand Hs00181036_m1; Applied Biosystems) were used. Each sample was run in duplicate, and the transcript quantity was normalized to the mRNA level of cyclophilin A (4326316E; Applied Biosystems).

Double-staining protocol. Aliquots of approximately 100 hand-picked islets were washed 2 times in $1 \mathrm{ml}$ of PBS, centrifuging for 2 minutes at $312 \mathrm{~g}$ at room temperature. Then, in order to asses the presence of TCF7L2 protein, the islets were incubated with $1 \mathrm{ml}$ of $10 \%$ normal blocking serum in $1 \mathrm{ml}$ of normal blocking serum (NBS; Santa Cruz Technologies) at $10 \%$ for 20 minutes at room temperature to suppress nonspecific binding of IgG. At the end, the islets were washed 2 times in PBS and incubated either with primary antibody for 60 minutes, then incubated for 45 minutes with fluorochrome-conjugated specific secondary antibody. The islets were washed 2 times in PBS, resuspended in $0.5 \mathrm{ml}$ of PBS and $2.5 \mu \mathrm{l}$ of $5 \mathrm{mmol} / \mathrm{l}$ RhodZin solution (Invitrogen) was added for 60 minutes at $37^{\circ} \mathrm{C}$. At the end of incubation period, the cells were examined using a fluorescence microscope with appropriate filters.

Arrest-In Transfection, which is a polymeric formulation developed and optimized for highly efficient delivery of plasmid into the nucleus of suspension cells in the presence of serum-containing medium, was purchased from Open Biosystems. Full ORF expression Clone (RZPD, Germany) for TCF7L2. Transfection was performed by adapting the manufacturer's instructions to human pancreatic islets. Briefly, the islets obtained from 3 pancreata were washed 12 times in Krebs-Ringer bicarbonate and exposed for 10 minutes in Krebs without $\mathrm{Ca}^{2+}$ and $\mathrm{Mg}^{2+}$ in order to gently disaggregate the sample. At the end of incubation time, 400 islets for each point studied were resuspended in $800 \mu$ l of M199 containing adult bovine serum and added in each well. Then, for each well in transfection, $2 \mu \mathrm{g}$ of plasmid was diluted into $100 \mu \mathrm{l}$ of M199 (step A), and $20 \mu \mathrm{g}$ of Arrest-In solution in $100 \mu$ l of M199 (step B). Then step A and step B were mixed rapidly and incubated for 10 minutes at room temperature to form the transfection complexes (step C). Finally, for each well containing islets, $200 \mu \mathrm{l}$ of step C was added and incubated for 72 hours in a $\mathrm{CO}_{2}$ incubator at $37^{\circ} \mathrm{C}$. After 72 hours incubation, the well volume was doubled $(2 \mathrm{ml})$ with M199 culture medium and the samples left in the incubator for an additional 48 hours. At the end of incubation period, islet function, viability, transfection efficiency, and gene expression were evaluated.

\section{Statistics}

We estimated the effect of genetic variants (risk versus non-risk genotypes) on the risk of developing T2D using Kaplan-Meier survival curves. The ORs for risk of developing T2D were calculated using logistic regression analyses adjusted for age at entry and time of follow-up, BMI, sex, and family history of diabetes. To test differences between group means (risk versus non-risk genotype), we used analysis of covariance (ANCOVA) adjusted for age, BMI, sex (when appropriate), and diabetes status (TCF7L2 mRNA). Variables that were not normally distributed (insulin, insulinogenic index, disposition index, area under insulin and glucose curve, basal hepatic glucose production) were log-transformed for analyses. Spearman's rank-order correlations controlled for age, BMI, sex, and time differences were used to assess associations of insulin response to IVGTTs and OGTTs between risk and non-risk genotypes, as well as association of TCF7L2 mRNA with insulin gene mRNA, total insulin content, and insulin and glucagon release. A generalized estimating equation (GEE) procedure was used to test differences in diabetes-related phenotypes over time separately in those who developed (converters) and those who did not develop (nonconverters) T2D adjusted for age, BMI, sex, and family relationships (44). In the GEE, the SEM were adjusted for repeated measurements in the same person with a robust variance estimator (45). The final measurement for converters was made at the visit when diabetes was diagnosed and for nonconverters at the last visit in the study.

All statistical analyses were performed with Statistical Package for the Social Sciences version 14.0 (SPSS), Number Crunching Statistical Systems version 2005 (NCSS), and STATA version 8. A 2-tailed $P$ value of 0.05 was considered statistically significant.

\section{Acknowledgments}

These studies were supported by grants from the Swedish Research Council, including a Linné grant (No. 31475113580), the Swedish Heart and Lung Foundation, the Swedish Diabetes Research Society, Diabetes Programme at the Lund University, the Finnish Diabetes Research Society, the Sigrid Juselius Foundation, the Påhlsson Foundation, the Crafoord Foundation, Folkhälsan Research Foundation, Novo Nordisk Foundation, the European Union (Integrated Project EuroDia LSHMCT-2006-518153 in Framework Programme 6 of the European Community), and the European Foundation for the Study of Diabetes/Pfizer Resource Award.

Received for publication October 20, 2006, and accepted in revised form May 22, 2007.

Address correspondence to: Valeriya Lyssenko, Department of Clinical Sciences, Diabetes and Endocrinology, Lund University, CRC, University Hospital Malmö, 20502 Malmö, Sweden. Phone: 46-40391214; Fax: 46-40-391222; E-mail: Valeri.Lyssenko@med.lu.se.
1. Gloyn, A.L., et al. 2003. Large-scale association studies of variants in genes encoding the pancreatic beta-cell KATP channel subunits Kir6.2 (KCNJ11) and SUR1 (ABCC8) confirm that the KCNJ11 E23K variant is associated with type 2 diabetes. Diabetes.
52:568-572.

2. Altshuler, D., et al. 2000. The common PPARgamma Pro12Ala polymorphism is associated with decreased risk of type 2 diabetes. Nat. Genet. 26:76-80.

3. Florez, J.C., et al. 2004. Haplotype structure and genotype-phenotype correlations of the sulfonylurea receptor and the islet ATP-sensitive potassium channel gene region. Diabetes. 53:1360-1368.

4. Grant, S.F., et al. 2006. Variant of transcription factor 7-like 2 (TCF7L2) gene confers risk of type 2 
diabetes. Nat. Genet. 38:320-323.

5. Groves, C.J., et al. 2006. Association analysis of 6,736 U.K. subjects provides replication and confirms TCF7L2 as a type 2 diabetes susceptibility gene with a substantial effect on individual risk. Diabetes. 55:2640-2644.

6. Florez, J.C., et al. 2006. TCF7L2 polymorphisms and progression to diabetes in the Diabetes Prevention Program. N. Engl. J. Med. 355:241-250.

7. Damcott, C.M., et al. 2006. Polymorphisms in the transcription factor 7-like 2 (TCF7L2) gene are associated with type 2 diabetes in the Amish: replication and evidence for a role in both insulin secretion and insulin resistance. Diabetes. 55:2654-2659.

8. Saxena, R., et al. 2006. Common single nucleotide polymorphisms in TCF712 are reproducibly associated with type 2 diabetes and reduce the insulin response to glucose in nondiabetic individuals. Diabetes. 55:2890-2895.

9. Zhang, C., et al. 2006. Variant of transcription factor 7-like 2 (TCF7L2) gene and the risk of type 2 diabetes in large cohorts of U.S. women and men. Diabetes. 55:2645-2648.

10. Cauchi, S., et al. 2006. Transcription factor TCF7L2 genetic study in the french population: expression in human beta-cells and adipose tissue and strong association with type 2 diabetes. Diabetes. 55:2903-2908.

11. Scott, L.J., et al. 2006. Association of transcription factor 7-like 2 (TCF7L2) variants with type 2 diabetes in a Finnish sample. Diabetes. 55:2649-2653.

12. Prunier, C., Hocevar, B.A., and Howe, P.H. 2004. Wnt signaling: physiology and pathology. Growth Factors. 22:141-150.

13. Yi, F., Brubaker, P.L., and Jin, T. 2005. TCF-4 mediates cell type-specific regulation of proglucagon gene expression by beta-catenin and glycogen synthase kinase-3beta. J. Biol. Chem. 280:1457-1464.

14. Berglund, G., et al. 2000. Long-term outcome of the Malmo preventive project: mortality and cardiovascular morbidity. J. Intern. Med. 247:19-29.

15. Lyssenko, V., et al. 2005. Genetic prediction of future type 2 diabetes. PloS Med. 2:e345.

16. Helgason, A., et al. 2007. Refining the impact of TCF7L2 gene variants on type 2 diabetes and adaptive evolution. Nat. Genet. 39:218-225.

17. Tripathy, D., et al. 2004. Parallel manifestation of insulin resistance and beta cell decompensation is compatible with a common defect in Type 2 diabetes. Diabetologia. 47:782-793.

18. Carlsson, A., Sundkvist, G., Groop, L., and Tuomi, T. 2000. Insulin and glucagon secretion in patients with slowly progressing autoimmune diabetes (LADA). J. Clin. Endocrinol. Metab. 85:76-80.
19. Ward, W.K., Bolgiano, D.C., McKnight, B., Halter, J.B., and Porte, D., Jr. 1984. Diminished B cell secretory capacity in patients with noninsulin-dependent diabetes mellitus. J. Clin. Invest. 74:1318-1328.

20. Meier, J.J., Deacon, C.F., Schmidt, W.E., Holst, J.J., and Nauck, M.A. 2007. Suppression of glucagon secretion is lower after oral glucose administration than during intravenous glucose administration in human subjects. Diabetologia. 50:806-813.

21. Nauck, M., Stockmann, F., Ebert, R., and Creutzfeldt, W. 1986. Reduced incretin effect in type 2 (non-insulin-dependent) diabetes. Diabetologia. 29:46-52.

22. Machicao, F., et al. 2006. Association of variants of the transcription factor 7 like 2 (TCF7L2) gene are associated with impaired insulin secretion, but not with impaired incretin secretion in individuals without diabetes [abstract]. Diabetes Care. 23:72.

23. Sladek, R., et al. 2007. A genome-wide association study identifies novel risk loci for type 2 diabetes. Nature. 445:881-885.

24. Diabetes Genetic Initiative of Broad Institute of Harvard and MIT, Lund University, and Nova rtis Institutes for BioMedical Research, et al. 2007. Genome-wide association analysis identifies loci for type 2 diabetes and triglyceride levels. Science. 316:1331-1336.

25. Zeggini, E., et al. 2007. Replication of genome-wide association signals in UK samples reveals risk loci for type 2 diabetes. Science. 316:1336-1341.

26. Scott, L.J., et al. 2007. A genome-wide association study of type 2 diabetes in Finns detects multiple susceptibility variants. Science. 316:1341-1345.

27. Steinthorsdottir, V., et al. 2007. A variant in CDKAL1 influences insulin response and risk of type 2 diabetes. Nat. Genet. 39:770-775.

28. Cauchi, S., et al. 2006. TCF7L2 variation predicts hyperglycemia incidence in a French general population: the data from an epidemiological study on the Insulin Resistance Syndrome (DESIR) study. Diabetes. 55:3189-3192.

29. Fehmann, H.C., Goke, R., and Goke, B. 1995. Cell and molecular biology of the incretin hormones glucagon-like peptide-I and glucose-dependent insulin releasing polypeptide. Endocr. Rev. 16:390-410.

30. Nauck, M.A., et al. 1993. Preserved incretin activity of glucagon-like peptide 1 [7-36 amide] but not of synthetic human gastric inhibitory polypeptide in patients with type-2 diabetes mellitus. J. Clin. Invest. 91:301-307.

31. Unger, R.H., and Orci, L. 1975. The essential role of glucagon in the pathogenesis of diabetes mellitus. Lancet. 1:14-16.

32. Granhall, C., Rosengren, A.H., Renstrom, E., and Luthman, H. 2006. Separately inherited defects in insulin exocytosis and beta-cell glucose metabolism contribute to type 2 diabetes. Diabetes. 55:3494-3500.

33. Parton, L.E., et al. 2006. Limited role for SREBP$1 \mathrm{c}$ in defective glucose-induced insulin secretion from Zucker diabetic fatty rat islets: a functional and gene profiling analysis. Am. J. Physiol. Endocrinol. Metab. 291:E982-E994.

34. Rulifson, I.C., et al. 2007. Wnt signaling regulates pancreatic beta cell proliferation. Proc. Natl. Acad. Sci. U. S. A. 104:6247-6252.

35. Eriksson, K.F., and Lindgarde, F. 1990. Impaired glucose tolerance in a middle-aged male urban population: a new approach for identifying highrisk cases. Diabetologia. 33:526-531.

36. Eriksson, K.F., and Lindgarde, F. 1991. Prevention of type 2 (non-insulin-dependent) diabetes mellitus by diet and physical exercise. The 6-year Malmo feasibility study. Diabetologia. 34:891-898.

37. Groop, L., et al. 1996. Metabolic consequences of a family history of NIDDM (the Botnia study): evidence for sex-specific parental effects. Diabetes. 45:1585-1593.

38. Del Guerra, S., et al. 2005. Functional and molecular defects of pancreatic islets in human type 2 diabetes. Diabetes. 54:727-735.

39. Lupi, R., et al. 2002. Lipotoxicity in human pancreatic islets and the protective effect of metformin. Diabetes. 51(Suppl. 1):S134-S137.

40. Matthews, D.R., et al. 1985. Homeostasis model assessment: insulin resistance and beta-cell function from fasting plasma glucose and insulin concentrations in man. Diabetologia. 28:412-419.

41. Bergman, R.N., Phillips, L.S., and Cobelli, C. 1981. Physiologic evaluation of factors controlling glucose tolerance in man: measurement of insulin sensitivity and beta-cell glucose sensitivity from the response to intravenous glucose. J. Clin. Invest. 68:1456-1467.

42. Heding, L.G. 1966. A simplified insulin radioimmunoassay method. In Labelled proteins in tracer studies. L. Donato, G. Milhaud, and I. Sirchis, editors. Euratom. Brussels, Belgium. 345-350.

43. Tang, K., et al. 1995. Matrix-assisted laser desorption/ionization mass spectrometry of immobilized duplex DNA probes. Nucleic Acids Res. 23:3126-3131.

44. Lyssenko, V., et al. 2005. Predictors of and longitudinal changes in insulin sensitivity and secretion preceding onset of type 2 diabetes. Diabetes. 54:166-174.

45. Liang, K.Y., and Zeger, S.L. 1986. Longitudinal data analysis using generalized linear models. Biometrika. 73:13-22. 